\title{
Feasibility of an 8-item questionnaire for early diagnosis of inflammatory bowel disease in primary care
}

Chmiel, Corinne ; Vavricka, Stephan R ; Hasler, Susann ; Rogler, Gerhard ; Zahnd, Nadine ; Schiesser, Salomé ; Tandjung, Ryan ; Scherz, Nathalie ; Rosemann, Thomas ; Senn, Oliver

Abstract: Aims Diagnosis of inflammatory bowel disease (IBD) is often associated with a diagnostic delay. Although faecal calprotectin is a helpful screening tool, the widespread use in primary care (PC) may not be appropriate due to the low prevalence of IBD in this setting. To increase pretest probability for a positive calprotectin test, an 8-item questionnaire (CalproQuest) was tested for its feasibility and acceptability in PC. Methods Population: PC patients with unspecific gastrointestinal complaints for at least 2 weeks. The CalproQuest consists of four major and four minor questions specific for IBD. It is considered positive if greater than or equal to two major or one major and two minor criteria are positive. Primary outcome: feasibility of CalproQuest, secondary outcome: patient's acceptance of stool sampling. Results Of 95 patients with a complete CalproQuest 52 (54.7\%) were positive, $39(41.1 \%)$ fulfilled two major and $13(13.7 \%)$ one major and greater than or equal to two minor criteria. Twenty-seven general practitioners completed $83(87.4 \%)$ questionnaires on feasibility which was assessed positive. Eighty-two patients $(86.3 \%)$ completed questionnaires on acceptance which was high. Conclusion The CalproQuest is a feasible instrument for assessing IBD in PC. Further prospective studies concerning validity and cost effectiveness of a combined use with the calprotectin test in this setting are necessary.

DOI: https://doi.org/10.1111/jep.13046

Posted at the Zurich Open Repository and Archive, University of Zurich ZORA URL: https://doi.org/10.5167/uzh-157255

Journal Article

Accepted Version

Originally published at:

Chmiel, Corinne; Vavricka, Stephan R; Hasler, Susann; Rogler, Gerhard; Zahnd, Nadine; Schiesser, Salomé; Tandjung, Ryan; Scherz, Nathalie; Rosemann, Thomas; Senn, Oliver (2019). Feasibility of an 8-item questionnaire for early diagnosis of inflammatory bowel disease in primary care. Journal of Evaluation in Clinical Practice, 25(1):155-162.

DOI: https://doi.org/10.1111/jep.13046 
Full Title: Feasibility of an 8-item-questionnaire for early diagnosis of inflammatory bowel disease in primary care

Short Title: Early diagnosis of inflammatory bowel

Corinne Chmiel ${ }^{a}$, Stephan R. Vavricka ${ }^{b}$, Susann Hasler ${ }^{a}$, Gerhard Rogler ${ }^{b}$, Nadine Zahnd ${ }^{b}$, Salomé Schiesser a , Ryan Tandjung a , Nathalie Scherz ${ }^{a}$, Thomas Rosemann a, Oliver Senn a

a Institute of Primary Care, University and University Hospital of Zurich, Switzerland

b Department of Gastroenterology and Hepatology, University Hospital Zürich, 8091 Zürich, Switzerland.

${ }^{*}$ Corresponding author:

Institute of Primary Care

UniversitätsSpital Zürich

Pestalozzistrasse 24

CH-8091 Zürich

Switzerland

phone: +41442553983

fax: +41442559097

email: corinne.chmiel@usz.ch 


\section{ABSTRACT}

\section{Aims}

Diagnosis of inflammatory bowel disease (IBD) is often associated with a diagnostic delay. Although fecal calprotectin is a helpful screening tool, the widespread use in primary care (PC) may not be appropriate due to the low prevalence of IBD in this setting. To increase pretest-probability for a positive calprotectin test, an 8-itemquestionnaire (CalproQuest) was tested for its feasibility and acceptability in PC.

\section{Methods}

Population: PC patients with unspecific gastrointestinal complaints for at least 2 weeks. The CalproQuest consists of 4 major and 4 minor questions specific for IBD. It is considered positive if $\geq 2$ major or 1 major and 2 minor criteria are positive. Primary outcome: feasibility of CalproQuest, secondary outcome: patient's acceptance of stool sampling.

\section{Results}

Of 95 patients with a complete CalproQuest 52 (54.7\%) were positive, 39 (41.1\%) fulfilled 2 major and $13(13.7 \%) 1$ major and $\geq 2$ minor criteria. 27 general practitioners completed $83(87.4 \%)$ questionnaires on feasibility which was assessed positive. 82 patients $(86.3 \%)$ completed questionnaires on acceptance which was high.

\section{Conclusion}

The CalproQuest is a feasible instrument for assessing IBD in PC. Further prospective studies concerning validity and cost-effectiveness of a combined use with the calprotectin test in this setting are necessary.

Trial registration number: ISRCTN66310845 


\section{KEYWORDS}

Inflammatory Bowel Disease

Crohn's disease

Ulcerative colitis

Early Diagnosis

Diagnostic Delay

Calprotectin

Feasibility

Primary Care

General Practitioner 


\section{INTRODUCTION}

Abdominal pain is one of the most frequent symptoms in primary care ${ }^{1,2}$. In the US, where respective data have been collected, 2.5 million consultations due to abdominal pain were recorded per year ${ }^{3}$. General practitioners (GP) often face the diagnostic challenge of identifying patients in need for further diagnostics and differentiating patients with inflammatory bowel disease (IBD) from functional disorders such as irritable bowel syndrome (IBS). Crohn's Disease (CD), ulcerative colitis (UC) and indeterminate colitis (IC) represent the three subtypes of IBD ${ }^{4}$. Estimated prevalence of IBD in the Swiss population is 205 cases per 100 '000 $(0.2 \%)^{5}$. Meanwhile, the prevalence of irritable bowel syndrome (IBS) in Europe and North America is estimated at $10-15 \%{ }^{6}$. Symptoms similar to IBS are frequently reported in patients before IBD is diagnosed ${ }^{7}$. Difficulties in recognizing early IBD, especially in primary care, lead to considerable diagnostic delay in $\mathrm{IBD}^{4}$, which has been shown to correlated with an increased risk of bowel stenosis and CD-related intestinal surgery ${ }^{8}$.

The gold diagnostic standard for IBD is endoscopy. However, not every patient with abdominal discomfort or pain in primary care can be investigated by means of an invasive endoscopic exam. Therefore, different non-invasive markers were developed to reduce the number of necessary endoscopies and hence to increase the likelihood of positive endoscopic results. Several studies, mainly originated in specialist care, have shown that fecal calprotectin reflects intestinal inflammation in patients with known IBD ${ }^{9},{ }^{10-12},{ }^{13-15}$. It has also been shown to differentiate IBD from IBS due to its good negative predictive value in discriminating IBD versus IBS, depending on the cutoff value used ${ }^{16},{ }^{17},{ }^{18},{ }^{19}$. Although calprotectin tests are easily accessible and reimbursed in Switzerland, this diagnostic test is not routinely performed in primary care. The reasons here fore have not yet been systematically elaborated; we assume the following considerations to play a role: a) the low prevalence of IBD in general practice. When analyzing the reasons for encounter in primary care, it becomes clear 
that digestive disorders are frequent complaints with a prevalence of $5-7 \%{ }^{20,21}$.

Considering the population-based prevalence of $10-15 \%$ of IBS compared with $0.2 \%$ of IBD, IBS is much more common in primary care. b) This consideration combined with the large amount of differential diagnosis for a positive calprotectin test besides IBD (esophagitis, gastritis, gastric ulcers, celiac disease, polyps and carcinomas, infections gastroenteritis, diverticulitis, microscopic and ischemic colitis, NSAR enteropathy, use of proton pump inhibitors, lactose intolerance) narrow the utility of the calprotectin test in this setting, besides $\mathrm{c}$ ) the relatively high costs (currently about 60 Euros).

Data assessing the diagnostic accuracy of the calprotectin in the primary care setting is scarce. In this setting, the pretest probability for a positive calprotectin is naturally low due to low prevalence of IBD. It is hence not astonishing that studies from primary care indicate a questionable diagnostic accuracy ${ }^{22-25}$. To increase pretest-probability for a positive calprotectin test and hence to increase its utility in the primary care setting, an 8-item-questionnaire (CalproQuest) was tested for its feasibility in primary care.

\section{METHODS AND ANALYSIS}

\section{Ethics, trial registration, informed consent and funding}

- Ethics: The study protocol was approved by the Ethics Committee of the Kanton Zurich (reference KEK-ZH-number 2013-0516).

- The study protocol conforms to the ethical guidelines of the 1975 Declaration of Helsinki as reflected in a prior approval by the institution's human research committee.

- $\quad$ Trial registration number: ISRCTN66310845.

- Written, informed consent was obtained from each patient included in the study.

- Fundig: This project is supported by grants from the IBDnet, Swiss Research and Communication Network on Inflammatory Bowel Disease, and the 
"Gottfried und Julia Bangerter-Rhyner-Stiftung", fund of the Swiss Academy of Medical Sciences. The funding sources had no role in the design of this study and will not have any role during its execution, analyses, interpretation of the data, or decision to submit results.

\section{Study design}

This study is a part of the prospective diagnostic ALERT trial (VAlidation of an 8-itemquestionnaire predictive for a positive caLprotectin tEst and Real-life implemenTation in primary care to reduce diagnostic delay in inflammatory bowel disease), consisting of two independent parts $A$ and $B$, conducted by gastroenterologists $(A)$ and GPs $(B)$. The details of the study design, including recruitment of patients and physicians, administration of patient records, informed consent and confidentiality have been published previously ${ }^{26}$.. Patients included in the study presented at their GP because of on-going unspecific gastrointestinal symptoms for at least two weeks (abdominal pain, bloating, stool irregularities, diarrhea). The study design and including the study flow is shown in Figure 1.

\section{Inclusion and exclusion criteria}

Inclusion criteria:

- $\quad 218$ years

- GP visit due to on-going unspecific gastrointestinal symptoms (abdominal pain, bloating, stool irregularities, diarrhea) for at least two weeks

- No earlier diagnostic procedures (endoscopy) for the current episode

- Informed consent

Exclusion criteria: 
- $\quad<18$ years

- Known further/other abdominal pathologies as e.g. cancer

- Previous abdominal surgeries

- Treatment with steroids (topical and/or oral) and/or amino salicylates within 30 days prior inclusion into this study

- Endoscopic examination within 3 years prior screening

Procedure (see also Figure 1)

- Patients were subjected to CalproQuest.

- Patients obtained fecal samples to measure calprotectin levels. Besides the possible diagnostic utility concerning the patient's complaints, the calprotectin was measured also in order to test for patient acceptance of stool sampling. No statement is possible concerning the validation of the CalproQuest with the calprotectin measures due to under powering.

- Patients completed the questionnaire on acceptance of stool sampling and physicians completed the questionnaire on feasibility of CalproQuest in daily practice.

- According to the current standard of care, patients with calprotectin levels $\geq 50 \mu \mathrm{g} / \mathrm{g}$ were referred to a gastroenterologist for endoscopic examination. It was at the discretion of the GP to follow this advice. The GP was informed about results of the endoscopy and he forwarded these results to the study center.

\section{CalproQuest}

CalproQuest is an 8-item IBD-questionnaire consisting of 4 major and 4 minor questions specific for IBD (Table 1). The CalproQuest is considered positive, if $\geq 2$ major criteria or 1 major criterion and 2 minor criteria are answered positively. We assumed that a positive CalproQuest result might predict calprotectin levels $\geq 50 \mu \mathrm{g} / \mathrm{g}$. 
Calprotectin levels above $50 \mu \mathrm{g} / \mathrm{g}$ are indicative for on-going intestinal inflammation and call for further endoscopic examination.

\section{Fecal Calprotectin}

Fecal calprotectin levels were measured at the University Hospital Zurich. Specimens from outpatients were sent to the laboratory by post. The calprotectin test is called EliA calprotectin (Thermo Fisher Scientific) and uses the FEIA method (fluorescence enzyme immunoassay) on a fully automated system called Phadia 100. The EliA calprotectin Wells are coated with monoclonal antibodies to calprotectin. If present in the patient's specimen, calprotectin binds to the coated antibodies. After washing away non-bound components, enzyme-labeled antibodies against human calprotectin (EliACalprotectin Conjugate) are added to form a calprotectin-conjugate complex. After incubation, non-bound conjugate is washed away and the bound complex is incubated with a development solution. After stopping the reaction, the fluorescence in the reaction mixture is measured. The higher the response value, the more calprotectin is present in the specimen. To evaluate test results, the response for patient samples is compared directly to the response for calibrators.

\section{Questionnaires}

The contents of the physician's questionnaire on feasibility and acceptance of CalproQuest in primary care and the patient questionnaire on acceptance of stool sampling are provided within the Figures 2 and 3.

\section{Primary and secondary outcomes}

Primary outcome: Feasibility of CalproQuest in daily primary care practice. Secondary outcome: Patient-reported acceptance of stool sampling. 


\section{Statistical analysis}

Likert-bar plots visualize feasibility of the CalproQuest and the patient-reported acceptance. For the comparison of symptoms of patients with a positive or negative calprotectin a Chisquare-Test was performed. $p<0.05$ is considered statistically significant. Statistical analysis was performed with $R(R \text { version 3.3.2 })^{27}$.

\section{RESULTS}

\section{Population}

Recruitment of GP's started in October 2014 and ended in November 2016.

Recruitment was performed by means of information events as well as mailings and personal contacts of the involved team. Therefore, no actual non-responder list was compiled. The study flow and Consort statement is shown in Figure 1 and Appendix 1. From 40 GPs, which were initially instructed, 35 finally agreed to participate and recruited patients. During study, one GP dropped out. The 34 GPs were mainly male $(25,73.5 \%)$, with a mean age of 49.4 years and working in practice since mean 12.4 years, mainly in group practices $(30,85.7 \%) .26(76.5 \%)$ GPs had used a calprotectin test before participating in the study.

The 34 GPs recruited between one and 7 patients (mean 3.1), in total 98 . From the 98 CalproQuests 95 were complete. 84 patients (mean (SD) age $38.0(14.5)$ years, $57.1 \%$ female) with complete CalproQuests underwent calprotectin testing. From the 95 CalproQuests 52 (54.7\%) were positive, 39 (41.1\%) fulfilled 2 major criteria and 13 $(13.7 \%)$ fulfilled one major and $\geq 2$ minor criteria. In 15 (15.8\%) fecal probe concentrations of $\geq 50 \mu \mathrm{g} / \mathrm{g}$ calprotectin were found. In $9(9.5 \%)$ the CalproQuest was likewise positive. The most common symptoms were abdominal pain $(78,80.4 \%)$ and 
diarrhea $(37,38.1 \%)$. The most common minor criteria were fatigue $(55,56.7 \%)$ and slime in feces $(28,28.9 \%)$. The distribution of symptoms did not show any significant difference between patients with calprotectin concentrations above or below $50 \mu \mathrm{g} / \mathrm{g}$ $(p=0.8896)$.

Since according to the study protocol it was not mandatory for the GP to send patients with a positive Calprotectin to endoscopic evaluation the data we received concerning endoscopic and histologic findings is far from complete. GPs sent the results of 5 endoscopies to the study center, of which 4 showed either no pathological findings or diverticulosis and/or polyps/adenomas, one showed evidence of CD.

\section{Primary outcome: Feasibility of CalproQuest in daily primary health care practice} 27 GPs completed or partially completed $83(87.4 \%)$ questionnaires consisting of seven items. The detailed distribution of answers concerning feasibility of the CalproQuest is shown in Figure 2. All items concerning feasibility were assessed positive on the four-level even-point Likert scale. Only few GPs stated that they prescribe calprotectin tests in patients with ongoing gastrointestinal symptom's regularly and therefore do not need the CalproQuest.

\section{Secondary outcome: Patient-reported acceptance of stool sampling}

82 patients $(86.3 \%)$ completed or partially completed the patient questionnaire consisting of a four-level even-point Likert scale with seven items. All patients understood the rationale of feces collection and the patient-reported acceptance of stool sampling was high (Figure 3). 


\section{DISCUSSION}

Our study showed that the CalproQuest is a feasible instrument for the assessment of IBD in primary care and that the patient reported acceptance of and understanding for stool sampling was high.

The rationale for the ALERT trial is the reduction of diagnostic delay in IBD patients. Since most patients present to their GP with unspecific abdominal complaints first, improving diagnostic procedures for diagnosing IBD patients in primary care is one of the most important starting points to reduce diagnostic delay. However, following factors render the optimal diagnostic procedure extremely challenging in primary care: reasons of encounter for digestive disorders are common (5-7\%) ${ }^{20,2128,29}$ but the prevalence of IBD extremely low $(0.2 \%)^{5}$, compared to a much higher prevalence of functional disorders $(10-15 \%)^{6}$. In this low prevalence setting, pretest probability for positive diagnostic test results such as the calprotectin are naturally low ${ }^{23}$. Since not all patients with unspecific gastrointestinal complaints can undergo invasive endoscopic examination, it is of utmost importance, that other non-invasive diagnostic procedures are developed to reduce morbidity and mortality of a diagnostic delay in IBD. To increase pretest-probability for a positive calprotectin test and hence to increase its utility in the primary care setting, an 8-item-questionnaire (CalproQuest) was tested for its feasibility in primary care.

Very few studies currently exist to compare our findings. Danese et al. published a 21 item questionnaire, which was developed by means of a systematic literature review in which CD specialists identified "red flags", i.e. symptoms or sings suggestive of $C^{30}$. Healthy as well as known CD patients were subjected to the questionnaire and had to recall symptoms. The questionnaire was able to successfully discriminate functional 
disorders from CD. This questionnaire however was not yet prospectively validated and not tested for feasibility, which seems necessary considering large content compared to the 7 items of the CalproQuest.

Contradictory to findings from other studies ${ }^{31}$, our study population showed a high acceptance concerning stool sampling, probably due to good communication skills of the GPs in ours study population. The diagnostic strategy of combining a questionnaire with fecal sampling to measure calprotectin levels therefore seems feasible in the Swiss primary care setting.

\section{Strengths and limitations}

In the primary care setting this is one of the few existing studies on the noninvasive assessment of IBD, almost none of the former studies are prospective and most originate from secondary and tertiary care ${ }^{32}$. To our knowledge, the ALERT trial is the first attempt of prospectively developing a questionnaire for the assessment of IBD in primary care.

The according to the sample size calculation targeted number of 80 patients assumed necessary for the feasibility testing in our study was more than achieved $(n=95)$. We abstained from restrictive inclusion criteria for the participation in the study. Therefore, patients represent the typical clientele with unspecific gastrointestinal complaints, which the GP is confronted with in daily practice. We therefore consider the study population to be representative. The distribution of participating GPs concerning age and gender was similar to the statistics of the Swiss Medical Association ${ }^{33}$, therefore generalizability can be assumed. Nevertheless, a selection bias of motivated GPs as well as patients cannot be neglected. 


\section{Conclusion}

The CalproQuest is a feasible instrument for the assessment of IBD in primary care.

Further prospective studies concerning the validity and cost-effectiveness of a combined use with the calprotectin test in this setting are necessary.

\section{ABBREVIATIONS}

Inflammatory Bowel Disease (IBD)

Crohn's Disease (CD)

Ulcerative colitis (UC)

Indeterminate colitis (IC)

Irritable bowel syndrome (IBS)

8-item-questionnaire to increase pretest-probability for a positive test result of the fecal

Calprotectin test (CalproQuest)

General Practitioner (GP)

\section{ETHICS and INFORMED CONSENT}

Ethics: The study protocol was approved by the Ethics Committee of the Kanton Zurich (reference KEK-ZH-number 2013-0516).

The study protocol conforms to the ethical guidelines of the 1975 Declaration of Helsinki as reflected in a prior approval by the institution's human research committee. Written, informed consent was obtained from each patient included in the study.

\section{FUNDING}

This project is supported by grants from the IBDnet, Swiss Research and

Communication Network on Inflammatory Bowel Disease, and the "Gottfried und Julia Bangerter-Rhyner-Stiftung", fund of the Swiss Academy of Medical Sciences. The 
funding sources had no role in the design of this study and will not have any role during its execution, analyses, interpretation of the data, or decision to submit results.

\section{COMPETING INTERESTS}

The authors declare that they have no competing interests.

\section{AUTHORS CONTRIBUTIONS}

TR, SV and GR were the initiators for this study. TR is the trial sponsor. TR, SV, GR and NZ developed the questionnaires. SH, RT, SM and TR organized the recruitment of the practices. SH, OS, TR and RT were involved in the development of the study protocol. SH wrote and revised the study protocol. CC analyzed the study data. CC, TR, NS and OS interpreted the study data. CC wrote and revised the final manuscript and all authors read revised and approved it. 


\section{REFERENCES}

1. Schappert SM. National Ambulatory Medical Care Survey: 1991 summary. Vital Health Stat 13. 1994(116):1-110.

2. Wallander MA, Johansson S, Ruigomez A, Garcia Rodriguez LA. Unspecified abdominal pain in primary care: the role of gastrointestinal morbidity. Int J Clin Pract. 2007;61(10):1663-1670.

3. Digestive diseases in the United States: Epidemiology and impact. NIH Publication No. 94-1447 https://doi.org/10.1016/0016-5085(95)90479-4. Accessed August 20th, 2017.

4. Vavricka SR, Spigaglia SM, Rogler G, et al. Systematic evaluation of risk factors for diagnostic delay in inflammatory bowel disease. Inflammatory bowel diseases. 2012;18(3):496-505.

5. Juillerat P, Pittet V, Bulliard JL, et al. Prevalence of Inflammatory Bowel Disease in the Canton of Vaud (Switzerland): A population-based cohort study. Journal of Crohn's \& colitis. 2008;2(2):131-141.

6. Guideline WGOG. Irritable bowel syndrome: a global perspective http://www.worldgastroenterology.org/assets/downloads/en/pdf/guidelines/20 irrita ble bowel syndrome.pdf. 2009.

7. Bercik $\mathrm{P}$, Verdu $E F$, Collins $\mathrm{SM}$. Is irritable bowel syndrome a low-grade inflammatory bowel disease? Gastroenterology clinics of North America. 2005;34(2):235-245, vi-vii.

8. Schoepfer AM, Dehlavi MA, Fournier N, et al. Diagnostic delay in Crohn's disease is associated with a complicated disease course and increased operation rate. The American journal of gastroenterology. 2013;108(11):1744-1753; quiz 1754.

9. Lin JF, Chen JM, Zuo JH, et al. Meta-analysis: fecal calprotectin for assessment of inflammatory bowel disease activity. Inflammatory bowel diseases. 2014;20(8):14071415.

10. Limburg PJ, Ahlquist DA, Sandborn WJ, et al. Fecal calprotectin levels predict colorectal inflammation among patients with chronic diarrhea referred for colonoscopy. The American journal of gastroenterology. 2000;95(10):2831-2837.

11. Roseth AG, Aadland E, Jahnsen J, Raknerud N. Assessment of disease activity in ulcerative colitis by faecal calprotectin, a novel granulocyte marker protein. Digestion. 1997;58(2):176-180.

12. Costa F, Mumolo MG, Ceccarelli L, et al. Calprotectin is a stronger predictive marker of relapse in ulcerative colitis than in Crohn's disease. Gut. 2005;54(3):364-368.

13. Schoepfer AM, Beglinger C, Straumann A, Trummler M, Renzulli P, Seibold F. Ulcerative colitis: correlation of the Rachmilewitz endoscopic activity index with fecal calprotectin, clinical activity, C-reactive protein, and blood leukocytes. Inflammatory bowel diseases. 2009;15(12):1851-1858.

14. Schoepfer AM, Beglinger C, Straumann A, et al. Fecal calprotectin correlates more closely with the Simple Endoscopic Score for Crohn's disease (SES-CD) than CRP, blood leukocytes, and the CDAI. The American journal of gastroenterology. 2010;105(1):162169.

15. D'Haens $G$, Ferrante $M$, Vermeire $S$, et al. Fecal calprotectin is a surrogate marker for endoscopic lesions in inflammatory bowel disease. Inflammatory bowel diseases. 2012;18(12):2218-2224.

16. van Rheenen PF, Van de Vijver E, Fidler V. Faecal calprotectin for screening of patients with suspected inflammatory bowel disease: diagnostic meta-analysis. $B m j$. 2010;341:c3369.

17. Tibble JA, Sigthorsson G, Foster R, Forgacs I, Bjarnason I. Use of surrogate markers of inflammation and Rome criteria to distinguish organic from nonorganic intestinal disease. Gastroenterology. 2002;123(2):450-460. 
18. Otten CM, Kok L, Witteman BJ, et al. Diagnostic performance of rapid tests for detection of fecal calprotectin and lactoferrin and their ability to discriminate inflammatory from irritable bowel syndrome. Clinical chemistry and laboratory medicine : CCLM / FESCC. 2008;46(9):1275-1280.

19. Schoepfer AM, Trummler M, Seeholzer P, Seibold-Schmid B, Seibold F. Discriminating IBD from IBS: comparison of the test performance of fecal markers, blood leukocytes, CRP, and IBD antibodies. Inflammatory bowel diseases. 2008;14(1):32-39.

20. Mansson J, Nilsson G, Strender LE, Bjorkelund C. Reasons for encounters, investigations, referrals, diagnoses and treatments in general practice in Sweden--a multicentre pilot study using electronic patient records. The European journal of general practice. 2011;17(2):87-94.

21. Moth G, Olesen F, Vedsted P. Reasons for encounter and disease patterns in Danish primary care: changes over 16 years. Scandinavian journal of primary health care. 2012;30(2):70-75.

22. Holtman GA, Lisman-van Leeuwen Y, Kollen BJ, et al. Diagnostic Accuracy of Fecal Calprotectin for Pediatric Inflammatory Bowel Disease in Primary Care: A Prospective Cohort Study. Ann Fam Med. 2016;14(5):437-445.

23. Holtman GA, Lisman-van Leeuwen $\mathrm{Y}$, Kollen BJ, et al. Challenges in diagnostic accuracy studies in primary care: the fecal calprotectin example. Bmc Family Practice. 2013;14.

24. Mowat C, Digby J, Strachan JA, et al. Faecal haemoglobin and faecal calprotectin as indicators of bowel disease in patients presenting to primary care with bowel symptoms. Gut. 2016;65(9):1463-1469.

25. Pavlidis P, Chedgy FJQ, Tibble JA. Diagnostic accuracy and clinical application of faecal calprotectin in adult patients presenting with gastrointestinal symptoms in primary care. Scandinavian journal of gastroenterology. 2013;48(9):1048-1054.

26. Hasler S, Zahnd N, Muller S, et al. VAlidation of an 8-item-questionnaire predictive for a positive caLprotectin tEst and Real-life implemenTation in primary care to reduce diagnostic delay in inflammatory bowel disease (ALERT): protocol for a prospective diagnostic study. BMJ Open. 2015;5(3):e007306.

27. R Development Core Team (2008). R: A language and environment for statistical computing. R Foundation for Statistical Computing V, Austria. ISBN 3-900051-07-0, URL http://www.R-project.org.

28. Chmiel C, Bhend H, Senn O, Zoller M, Rosemann T, study-group F. The FIRE project: a milestone for research in primary care in Switzerland. Swiss medical weekly. 2011;140:w13142.

29. Tandjung R, Hanhart A, Bartschi F, et al. Referral rates in Swiss primary care with a special emphasis on reasons for encounter. Swiss medical weekly. 2015;145:w14244.

30. Danese S, Fiorino G, Mary JY, et al. Development of Red Flags Index for Early Referral of Adults with Symptoms and Signs Suggestive of Crohn's Disease: An IOIBD Initiative. Journal of Crohn's \& colitis. 2015;9(8):601-606.

31. Lecky DM, Hawking MKD, McNulty CAM, Grp ES. Patients' perspectives on providing a stool sample to their GP: a qualitative study. Brit J Gen Pract. 2014;64(628):E684-E693.

32. Jellema $\mathrm{P}$, van Tulder MW, van der Horst HE, Florie J, Mulder CJ, van der Windt DA. Inflammatory bowel disease: a systematic review on the value of diagnostic testing in primary care. Colorectal disease : the official journal of the Association of Coloproctology of Great Britain and Ireland. 2011;13(3):239-254.

33. Hostettler S, Kraft E. FMH-Ärztestatistik 2014: Frauen- und Ausländeranteil nehmen kontinuierlich zu. Schweizerische Ärztezeitung. 2014;13:462-469. 


\section{FIGURE LEGENDS}

Figure 1 Study flow Feasibility of CalproQuest.

Legend: Neg=negative; Pos=positive. GP: General Practitioner.

Figure 2 Feasibility of CalproQuest in daily primary care practice.

Legend: Four-level Likert scale: 1 (Strongly disagree) to 4 (Strongly agree).

Figure 3 Patient-reported acceptance of stool sampling.

Legend: Four-level Likert scale: 1 (Strongly disagree) to 4 (Strongly agree). 


\section{TABLES}

Table 1: CalproQuest (8-item IBD questionnaire)

\begin{tabular}{|c|c|c|c|c|}
\hline Type & Criteria & $\begin{array}{l}\text { Yes } \\
\text { (1) }\end{array}$ & $\begin{array}{l}\text { No } \\
\text { (0) }\end{array}$ & Comment \\
\hline \multirow{4}{*}{ Major } & $\begin{array}{l}\text { Does the patient suffer from abdominal pain } \\
\text { at least } 3 \text { times a week for at least } 4 \text { weeks? }\end{array}$ & & & \\
\hline & $\begin{array}{l}\text { Does the patient suffer from diarrhea (more } \\
\text { than } 3 \text { bowel movements daily) for } 7 \\
\text { consecutive days? }\end{array}$ & & & \\
\hline & $\begin{array}{l}\text { Does the patient have diarrhea at night- } \\
\text { time/Does the patient awake from sleep } \\
\text { because of abdominal pain or diarrhea? }\end{array}$ & & & \\
\hline & Does the patient report bloody stool? & & & \\
\hline \multirow{4}{*}{ Minor } & $\begin{array}{l}\text { Does the patient report mucus in stool for } \\
\text { more than } 4 \text { weeks? }\end{array}$ & & & \\
\hline & $\begin{array}{l}\text { Does the patient report unwanted weight } \\
\text { loss ( } 5 \% \text { of normal body weight over } 6 \\
\text { months)? }\end{array}$ & & & \\
\hline & $\begin{array}{l}\text { Does the patient present with fever or report } \\
\text { fever over the last } 4 \text { weeks (Temperature }> \\
38^{\circ} \mathrm{C} \text { )? }\end{array}$ & & & \\
\hline & $\begin{array}{l}\text { Does the patient report fatigue over the last } \\
4 \text { weeks? }\end{array}$ & & & \\
\hline
\end{tabular}

